\title{
Influence of friction on granular segregation
}

\author{
Stephan Ulrich, ${ }^{*}$ Matthias Schröter, ${ }^{\dagger}$ and Harry L. Swinney \\ Center for Nonlinear Dynamics and Department of Physics, The University of Texas at Austin, Austin, Texas 78712, USA
}

(Received 24 June 2007; published 9 October 2007)

\begin{abstract}
Vertical shaking of a mixture of small and large beads can lead to segregation where the large beads either accumulate at the top of the sample, the so-called Brazil nut effect (BNE), or at the bottom, the reverse Brazil nut effect (RBNE). Here we demonstrate experimentally a sharp transition from the RBNE to the BNE when the particle coefficient of friction increases due to aging of the particles. This result can be explained by the two competing mechanisms of buoyancy and sidewall-driven convection, where the latter is assumed to grow in strength with increasing friction.
\end{abstract}

DOI: 10.1103/PhysRevE.76.042301

PACS number(s): 45.70.Mg, 81.40.Pq

\section{INTRODUCTION}

Segregation of particles in granular media is a common problem in the chemical and pharmaceutical industries and in materials processing. Several physical mechanisms have been found to lead to segregation [1,2], but their dependence on material parameters and especially the role of friction [3-5] are not well understood. Here we report experimental results on the segregation of a vertically shaken mixture of $2.4 \mathrm{~mm}$ diameter brass spheres and $1.4 \mathrm{~mm}$ diameter glass spheres. Samples of new particles exhibit a reverse Brazil nut effect (RBNE) where all of the large particles accumulate at the bottom plate of the sample. However, after about $25 \mathrm{~h}$ of continuous shaking a sharp transition to a Brazil nut effect (BNE) occurs for unchanged driving conditions. Measurements show that the main effect of the aging is an increase of the coefficient of friction $\mu$. The initial state (RBNE) can be restored with an aggressive cleaning procedure which also decreases $\mu$. The cleaned sample shows the same transition to BNE during subsequent aging.

\section{EXPERIMENT}

The experimental setup is shown in Fig. 1. The particles are shaken in a $21 \mathrm{~mm} \times 21 \mathrm{~mm}$ square glass tube with a height of $150 \mathrm{~mm}$. The cell is mounted on an electromechanical shaker (Vibration Test Systems VG100c) and shaken sinusoidally with an amplitude $A$ and a fixed frequency $f=20 \mathrm{~Hz}$. The dimensionless shaking acceleration is $\Gamma=(2 \pi f)^{2} A / g$, where $g$ is the acceleration due to gravity. The sample consists of 74 brass (alloy 260) beads with $2.4 \mathrm{~mm}$ diameter $\left(\rho=8.4 \mathrm{~g} / \mathrm{cm}^{3}\right)$ and about 820 glass beads (Ceroglass GSR 14) with $1.4 \mathrm{~mm}$ diameter $\left(\rho=2.5 \mathrm{~g} / \mathrm{cm}^{3}\right)$.

Measurements of each segregation state were performed at both $\Gamma=2$ and 5. Before each measurement the beads were shaken for $2 \mathrm{~min}$ at the $\Gamma$ of the measurement; this time was found to be sufficient in Ref. [2] for the system to reach a stationary state, independent of the previous shaking history. The segregation state for each condition was determined by

\footnotetext{
*Present address: Institut für Theoretische Physik, Georg-AugustUniversität Göttingen, 37073 Göttingen, Germany.

†schroeter@chaos.utexas.edu
}

stopping the shaker and obtaining images from the top and bottom surface of the sample. All large particles visible in these images were then detected with image processing, allowing a quantitative characterization of the segregation (for details see Ref. [2]). After each set of measurements the beads were aged for one hour by shaking them constantly at $\Gamma=5$ before making a new set of measurements.

Table I shows that the aging of the particles is accompanied by an increase of $\mu$. Treating the particles for $10 \mathrm{~min}$ in an ultrasonic bath with a concentrated solution of Alconox powdered cleaner reduces $\mu$.

To exclude alternative causes for the observed transition we also measured the coefficient of restitution $e$ by dropping the particles from a height of $30 \mathrm{~cm}$ on a plate of the same material. For both cleaned and aged particles we measured $e=0.65 \pm 0.02$ for the brass and $e=0.89 \pm 0.01$ for the glass particles. The relative humidity (measured with a Honeywell HIH 3610 sensor) was $54 \pm 3 \%$ during the experiment and the fluctuations were not correlated with the transition. Cleaning the container and particles with soap and water did not change the BNE back to a RBNE. Also, pausing the aging process for up to $48 \mathrm{~h}$ did not influence the transition.
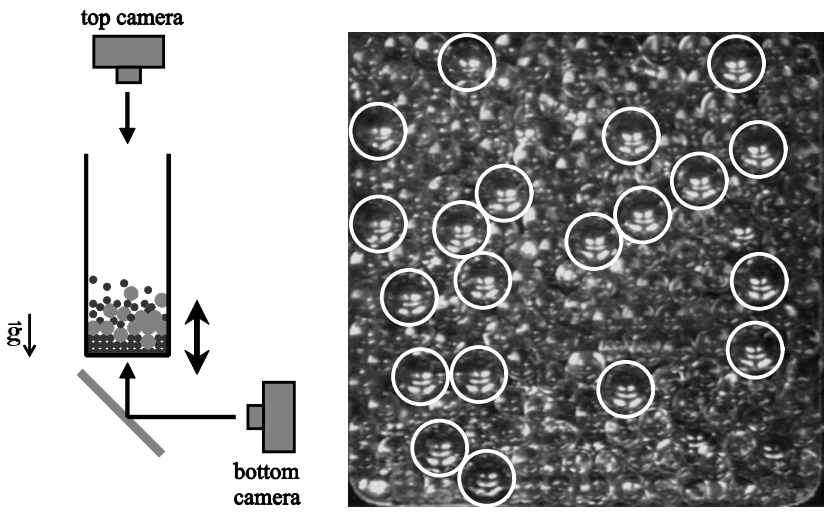

FIG. 1. Left: Experimental setup. The particles are shaken vertically in a square glass tube. Pictures are taken from the top and the bottom (using a mirror) of the granular sample. Right: An image of the bottom plate. All large particles detected by the image processing are marked with white circles. 
TABLE I. Static coefficients of friction $\mu$ of glass and brass spheres that were either freshly surface treated or aged by shaking for $28 \mathrm{~h}$. Measurements were performed with three particles glued to the bottom of a small toboggan. $\mu$ was determined from the angle when the toboggan started to slide on a brass or glass chute. This method has been found to yield similar results to measurements using a tribometer or based on the angle of repose [4]. Each value reported here is the average of five different sets of balls.

\begin{tabular}{lcc}
\hline \hline & "Cleaned" particles & Aged particles \\
\hline On a brass chute: & & \\
Brass sphere & $0.169 \pm 0.016$ & $0.196 \pm 0.009$ \\
Glass sphere & $0.151 \pm 0.003$ & $0.162 \pm 0.006$ \\
On a glass chute: & & \\
Brass sphere & $0.086 \pm 0.009$ & $0.111 \pm 0.003$ \\
Glass sphere & $0.089 \pm 0.006$ & $0.095 \pm 0.004$ \\
\hline \hline
\end{tabular}

\section{RESULTS AND DISCUSSION}

Figure 2(a) shows for new particles a sharp transition from the RBNE state to the BNE state after $25 \mathrm{~h}$ both at $\Gamma$ $=2$ and 5 . Continued shaking up to $180 \mathrm{~h}$ did not change the BNE observed. However, Figs. 2(b) and 2(c) demonstrate that the surface treatment described above resets the initial RBNE. Also the transition to the BNE state after about $25 \mathrm{~h}$ is reproducible.

This transition can be explained by the interaction of two segregation mechanisms: buoyancy-weight [5-10] and sidewall-driven convection [6,11-15]. Initially the dense brass particles sink to the bottom of the vibration-fluidized sample due to their buoyancy being smaller than their weight [16]. This mechanism does not depend strongly on the frictional properties of the beads as evidenced by its existence in simulations with frictionless particles [7].

The second segregation mechanism present in our setup and leading on its own to a BNE is sidewall-driven convec-

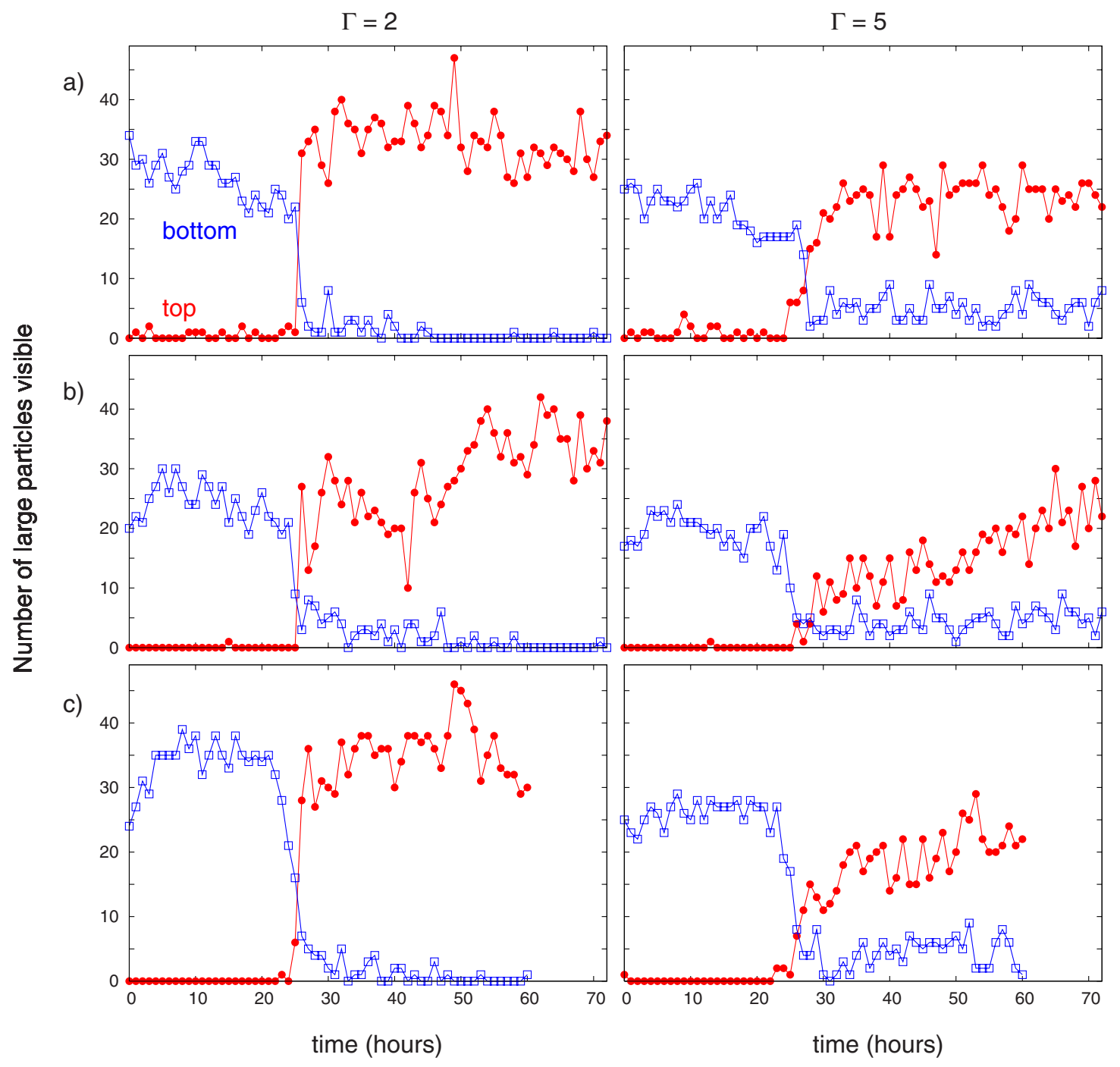

FIG. 2. (Color online) Numbers of large particles at the top and bottom surface of the sample measured at $\Gamma=2$ (left column) or 5 (right column). In both cases the segregation changes from a reverse Brazil nut state to a Brazil nut state after about $25 \mathrm{~h}$ of aging the particles. Experiment (a) started with new particles; the particles in (b) and (c) were treated in an alkaline ultrasound bath before the start of the experiment. These data were obtained for $f=20 \mathrm{~Hz}$. 
tion: the difference in packing density during the upward and downward motion of the container gives rise to a convection roll going downwards at the sidewalls and upwards in the center. Segregation occurs because the larger particles move upwards in the center but have a lower probability of entering the much thinner downstream layer at the container walls. An increase in $\mu$ can be expected to strengthen the convective motion $[17,18]$ and therefore also strengthen this segregation mechanism.

The transition at $\Gamma=2$ is steeper than the transition at $\Gamma$ $=5$. This can be explained by the increasing importance of a third mechanism, called thermal diffusion. Thermal diffusion describes the tendency of the larger particles to accumulate in a minimum of the granular temperature; its strength increases with increasing $\Gamma$ and leads to a RBNE state in shallow layers such as the ones considered here $[2,19,20]$.

\section{CONCLUSION}

The transition from RBNE to BNE can be explained by assuming that during the aging process $\mu$ and consequentially the sidewall-driven convection increase until this mechanism surmounts the initially dominant buoyancyweight mechanism. The sharpness of the observed transition indicates that minute changes in the coefficient of friction can have dramatic effects on the segregation behavior. This insight could be important for the industrial handling of granular matter.

\section{ACKNOWLEDGMENTS}

We thank W. D. McCormick and Yair Shokef for helpful discussions. This work was supported in part by Robert A. Welch Foundation Grant No. F-0805.
[1] A. Kudrolli, Rep. Prog. Phys. 67, 209 (2004).

[2] M. Schröter, S. Ulrich, J. Kreft, J. B. Swift, and H. L. Swinney, Phys. Rev. E 74, 011307 (2006).

[3] Y. Srebro and D. Levine, Phys. Rev. E 68, 061301 (2003).

[4] N. A. Pohlman, B. L. Severson, J. M. Ottino, and R. M. Lueptow, Phys. Rev. E 73, 031304 (2006).

[5] M. P. Ciamarra, M. D. De Vizia, A. Fierro, M. Tarzia, A. Coniglio, and M. Nicodemi, Phys. Rev. Lett. 96, 058001 (2006).

[6] T. Shinbrot and F. J. Muzzio, Phys. Rev. Lett. 81, 4365 (1998).

[7] N. Shishodia and C. R. Wassgren, Phys. Rev. Lett. 87, 084302 (2001).

[8] L. Trujillo, M. Alam, and H. J. Herrmann, Europhys. Lett. 64, 190 (2003).

[9] D. A. Huerta and J. C. Ruiz-Suárez, Phys. Rev. Lett. 92, 114301 (2004).

[10] G. Gutiérrez, O. Pozo, L. I. Reyes, R. Paredes, J. F. Drake, and
E. Ott, Europhys. Lett. 67, 369 (2004).

[11] J. B. Knight, H. M. Jaeger, and S. R. Nagel, Phys. Rev. Lett. 70, 3728 (1993).

[12] J. Duran, T. Mazozi, E. Clément, and J. Rajchenbach, Phys. Rev. E 50, 5138 (1994).

[13] T. Pöschel and H. J. Herrmann, Europhys. Lett. 29, 123 (1995).

[14] Y. Lan and A. D. Rosato, Phys. Fluids 9, 3615 (1997).

[15] M. E. Möbius, X. Cheng, P. Eshuis, G. S. Karczmar, S. R. Nagel, and H. M. Jaeger, Phys. Rev. E 72, 011304 (2005).

[16] D. A. Huerta, V. Sosa, M. C. Vargas, and J. C. Ruiz-Suárez, Phys. Rev. E 72, 031307 (2005).

[17] J. B. Knight, Phys. Rev. E 55, 6016 (1997).

[18] E. L. Grossman, Phys. Rev. E 56, 3290 (1997).

[19] D. Serero, I. Goldhirsch, S. H. Noskowicz, and M. -L. Tan, J. Fluid Mech. 554, 237 (2006).

[20] V. Garzó, Europhys. Lett. 75, 521 (2006). 